\title{
Elaboração de medidas preventivas para o controle de infecção cruzada em exames de eletroencefalograma
}

\section{Elaboration of preventive measures for the controlo of cross infection in electroencephalogram exams}

\author{
Amanda dos Santos Cecilio' • lone Corrêa² • Maria Justina Dalla Bernardina Felippe ${ }^{3}$
}

\begin{abstract}
RESUMO
Objetivo: Avaliar fatores de risco de disseminação de microrganismos relacionados com dermatoses do couro cabeludo para elaboração de medidas preventivas e controle de infecção cruzada em exames de eletroencefalograma. Método: Revisão Integrativa com estratégia de busca nas bases de dados: CINAHL, Embase, Pubmed, Scopus e Wos. O levantamento foi durante janeiro a fevereiro de 2018. Como critérios de inclusão utilizaram-se estudos que abordassem a temática da correlação entre exame de eletroencefalograma e infecção cruzada, em periódicos e textos disponíveis na íntegra nacionais ou internacionais nos idiomas português, espanhol ou inglês indexado e sem delimitação temporal. Resultados: Foram identificados 936 artigos e foram inseridos quatro artigos para o presente estudo, aos quais a abrasão realizada no couro cabeludo do paciente, os eletrodos e suas extensões que entram em contato com a pele, a falha no procedimento de desinfecção dos equipamentos, a falta de controles de higienização, a falta de treinamento com a equipe envolvida no processo de trabalho e a falta de protocolos a serem seguidos, são fatores de risco que requerem medidas/ações para promover motivação e capacitação com responsabilidade profissional e social. Conclusão: Os riscos de adquirir infecções em serviços de saúde são elevados e em grande parte poderiam ser evitados por meio da adesão dos profissionais de saúde e realização de medidas/ações de prevenção e controle de infecção promovem a segurança do paciente. Diante de tal necessidade, foi elaborado mapeamento de processo do serviço de neurologia diagnóstica, contendo normas e rotinas, procedimento operacional padrão, indicadores e disponibilização do e-book.

Descritores: Dermatoses de Couro Cabeludo; Eletroencefalograma; Desinfecção; Instituições de Saúde; Infecção Cruzada.
\end{abstract}

\begin{abstract}
Objective:To evaluate risk factors to microorganisms' dissemination related to dermatoses of the scalp. To perform measures of prevention and control of cross infection in electroencephalogram exams. Method: Integrative revision with search strategy in databases: CINAHL, Embase, PubMEd, Scopus and Wos. The search was carried out from January to February 2018. As inclusion criteria were researches by topic electroencephalogram and cross-infection, in national and international journals and texts available in Portuguese, Spanish or English indexed and without temporal delimitation. Results: A total of 936 articles were identified. Four articles were inserted for the present study, to which the abrasion carried out on the patient's scalp, the electrodes and their extensions that come in contact with the skin, the failure in the disinfection of equipment, lack of sanitation controls, lack of training with the staff involved in the work process and lack of protocols to be followed, are risk factors that require measures/ actions to promote motivation and empowerment with responsibility professional and social. Conclusion:The risks of acquiring infections in health services are high and much of them could be avoided through the adhesion of health professionals. The implementation of measures and actions of prevention and infection control promote patient safety. In view of this need, a process mapping of the diagnostic neurology service was developed, containing norms and routines, standard operating procedures, indicators and the e-book availability.
\end{abstract}

Keywords: Dermatoses of Scalp; Electroencephalogram; Disinfection; Health Institutions; Cross Infection. Faculdade UNINGA, Brasil (201I - 201 I). Mestrado Profissional em Enfermagem. Universidade Estadual Júlio de Mesquita Filho, UNESP, Botucatu, Brasil (2017 - 2019). Especialização em Cardiologia pela Faculdade Unyleya, Brasil (2019 - 2020). Enferm Mauricio de Oliveira, 453, Jardim Paraiso, CEP: 18.610 , 261, Botucatu, Brasil. Email: amanda.cecilio@unesp.b

2Gauricio de Oliveira, 453, Jardim Paraiso, CEP: 18.610,261, Botucatu, Brasil. Emaill amanda.cecilio@unesp.br doutorado em Farmacologia pela Universidade Estadual de Campinas (1995). Atualmente é professora doutora da Universidade Estadual Paulista Júlio de Mesquita Filho, cargo efetivo no Curso de Graduação em Enfermagem e no Programa de Pós Graduação - Mestrado Profissionalizante em Enfermagem e no Programa de Pós Graduaçăo - Mestrado Acadêmico e Doutorado pela FMB/UNESP, Tutora no Programa de Residência em enfermagem cuidados críticos. Tem experiência na área de Enfermagem, com ênfase em Saúde da Criança, atuando principalmente nos seguintes temas: cuidado a criançalfamilia, infecção relacionada a assistência a saúde, educação em saúde e segurança do paciente. Email: ione.correa@unesp.br Possui graduação em Enfermagem pela Universidade do Sagrado Coração (1983), especialização em administração hospitalar pela Faculdade São Camilo, mestrado em Fisiopatologia em Clínica Médica pela Universidade Estadual Paulista Júlio de Mesquita Filho (2000) Especialização em Nefrologia e em Gerenciamento do Serviço de Enfermagem, doutorado em Doenças Tropicais pela Universidade Estadual Paulista Júlio de Mesquita Filho (2005). Fez MBA em Auditoria de Serviços de Saúde pelas Faculdades Metropolitanas Unidas de São Paulo e Especialização Auditoria em Serviços de Saúde pela Uningá e Especialização em Acreditação pela Fundação Lucas Machado de Belo Horizonte. Atuou como Diretor Técnico Saúde III no Departamento de Auditoria Avaliação e Controle e como Diretor do Núcleo de Gestão Qualidade do Hospital das Clínicas da Faculdade de Medicina de Botucatu da Universidade Estadual Paulista aúlio de Mesquita Filho. Atualmente é Coordenador do Curso de Graduação
em Enfermagem da Faculdade Galileu em Botucatu e Coordenador do Curso de Graduação em Enfermagem da Faculdade Gran Tietê em Barra Bonita. Tem experiência na área de Enfermagem em Saúde do Adulto e Idoso, Pronto Socorro e Centro Cirúrgico, com ênfase em Administração Hospitalar e do Serviço de Enfermagem atuando principalmente nas seguintes áreas: Hemodiálise, Pronto Socorro e Terapia Intensiva, Enfermagem em Nefrologia, Gestão Hospitalar e Auditoria. Endereço para correspondência: Rua: Carlos Guadanini, 2348, Jardim Paraíso, CEP I8610-I 20. Botucatu, São Paulo-Brasil. Email: majudalla@@gmail.com 


\section{INTRODUÇÃO}

Segurança do Paciente (SP) é definida como um conjunto de ações que reduzem o risco de danos associados à assistência à saúde utilizando-se das melhores evidências disponíveis e visando promover uma assistência qualificada ${ }^{(1)}$.

A ocorrência de erros e eventos adversos (EA), a segurança do paciente passou a ser uma estratégia focada na prevenção, notificação e seguimento de danos causados aos pacientes (2).

"A International Classification for Patient Safety (ICPS) classifica erro e incidentes como, respectivamente: "uma falha em executar um plano de ação como pretendido ou como a aplicação de um plano incorreto" e Incidente, um evento ou circunstância que poderia ter resultado, ou resultou, em dano desnecessário ao paciente"(3).

Os incidentes podem ser assim classificados: Near Miss (aquele que não atingiu o paciente); incidente sem dano (evento que atingiu o paciente, porém não causou dano discernível); e incidente com dano, nomeado por evento adverso, (que resulta em dano ao paciente) $)^{(3)}$.

A infecção hospitalar é um evento adverso e segundo a Organização Mundial da Saúde (OMS), é causa que mais mata em serviços de saúde em todo mundo. Portanto, a prevenção, a notificação e o controle da infecção hospitalar é um grande desafio para os serviços de saúde ${ }^{(4)}$.

O termo infecção hospitalar foi substituído pelo termo Infecções Relacionadas à Assistência à Saúde (IRAS), levando-se em conta sua ampliação, já que sua ocorrência se dá em todos os estabelecimentos que prestam o cuidado e a assistência à saúde(4).

Assim, IRAS são definidas como qualquer infecção adquirida após a admissão do paciente em unidade prestadora de assistência à saúde, podendo manifestar-se durante a internação ou após a alta, desde que estejam associados com os procedimentos realizados nas unidades de atendimento à saúde ${ }^{(4)}$.

É fundamental que todos que coexistam nestes ambientes saibam que prevenir e controlar infecções são responsabilidade de todos visto que a ICPS destaca o frágil hábito de segurança, voltada à ocorrência de eventos adversos e sua relação com a IRAS (3,5-6).

Desta maneira, a Resolução $\mathrm{n}^{\circ} 36$ da Agência Nacional de Vigilância Sanitária (ANVISA), institui ações de promoção para a SP e melhoria da qualidade nos serviços de saúde como: a higienização das mãos (HM) e procedimentos clínicos seguros ${ }^{(7)}$.

Uma proporção considerável das IRAS pode ser evitada com a higienização das mãos. Os microrganismos associados à tais infecções são pertencentes à microbiota transitória (adquirida através dos contatos estabelecidos com pessoas colonizadas ou infectadas e/ou com objetos inanimados contaminados) ${ }^{(8-9)}$.

Desde 1986, o médico húngaro Ignez Philip Semmelweis, comprovou a íntima relação da febre puerperal com os cuidados médicos e notou que eles iam diretamente à sala de autópsia para a de obstetrícia e tinham um odor desagradável na mão. Demonstrou claramente por estudo experimental sobre este tema, que a higienização apropriada das mãos podia prevenir infecções puerperais e evitar mortes maternas ${ }^{(10)}$.

Assim, as mãos apresentam-se como o principal veículo de transmissão de infecções e devem ser higienizadas antes e após qualquer procedimento empregado na assistência ao paciente. A limpeza do ambiente é parte importante na prevenção e controle de infecções cruzadas, incluindo pisos, paredes, macas, cadeiras de rodas e mobília do quarto(11-12).

Com os avanços tecnológicos e as estratégias adotadas, a ocorrência de IRAS continua sendo uma grande ameaça à segurança do paciente, aumentando as taxas de morbimortalidade e gerando um alto custo de hospitalização, devido ao tempo prolongado de permanência do paciente no hospital (13).

Existem ainda as infecções comunitárias que são importantes nesse contexto, pois são aquelas constatadas ou em incubação no ato de admissão do paciente, desde que não relacionada com internação anterior no mesmo hospital. A infecção está associada com complicação ou extensão da infecção já presente na admissão(14).

Na prestação da assistência à saúde durante a realização de exames diagnósticos em ambientes público ou privado, pode ocorrer situações que expõem o paciente ao risco de IRAS (13), como por exemplo, na realização de eletroencefalograma (EEG).

O EEG surgiu como exame diagnóstico em 1929, descoberto por um psiquiatra alemão Hans Berger quando afirma que era possível captar as fracas correntes elétricas geradas no cérebro humano, sem abertura do crânio e mostrálas na forma de um registro de papel(15-16).

Mesmo com o avanço de outros métodos diagnósticos, permanece na linha de frente dos exames complementares em Neurologia, para diagnosticar alterações neurológicas como: epilepsia; suspeita de alterações da atividade cerebral; casos de alterações da consciência (desmaio ou coma); detecção de inflamações ou intoxicações cerebrais; complemento da avaliação de pacientes com doenças cerebrais (demência ou psiquiátrica) e protocolo de morte encefálica ${ }^{(15-16)}$.

É realizado por meio da colocação de eletrodos de prata e/ ou ouro no couro cabeludo e estão ligadas a um computador que registra as ondas elétricas do cérebro, é indolor e pode ser realizada em qualquer faixa etária ${ }^{(15-16)}$.

Utiliza-se uma pasta condutora que,além de fixá-los, permite a transmissão adequada dos sinais elétricos. Os materiais que auxiliam na realização do exame são: amplificadores, filtros, eletrodos específicos, pastas condutoras e caixa de eletrodos com vinte e três canais ${ }^{(15-16)}$.

A pasta condutora é atóxica e volátil, com contraindicação de permanecer aberta. A pasta é usada de forma coletiva para pacientes atendidos ambulatorial e/ou internados. Assim, a equipe de enfermagem deve se atentar aos cuidados durante - manuseio e preparo do paciente considerando o risco de contaminação do produto e, consequentemente, risco de infecção cruzadas. Existem também as lesões do couro cabeludo, que diferem em contagiosas ou não contagiosas, sendo um importante desencadeador de interferência no resultado do exame ${ }^{(17-19)}$.

São consideradas lesões contagiosas do couro cabeludo: Tinea Capitis; Escabiose e Pediculose. E lesões não contagiosas, mas que potencialmente podem propagar disseminação de microrganismos: Dermatite seborreica e Psoríase.

Sabendo dos riscos de infecção aos quais os pacientes submetidos ao exame de EEG podem estar expostos, é essencial a avaliação do profissional enfermeiro e sua equipe, para a limpeza e desinfecção dos eletrodos do EEG entre o uso de um paciente e outro. É essencial o cuidado com a pasta condutora e demais utensílios utilizados para realização do exame e investigação/avaliação do couro cabeludo. 
A Sistematização da Assistência de Enfermagem (SAE), a implantação do Processo de Enfermagem (PE) e o gerenciamento de riscos, possibilita ao enfermeiro identificar riscos relacionados à assistência a saúde e minimizar os efeitos causados por esses riscos, quando os mesmos ocorrem em ambiente hospitalar e exames hospitalares (20-2I) como por exemplo, no Serviço de Neurologia Diagnóstica (SND).

A revisão bibliográfica se faz necessário para avaliar os fatores de risco e propor medidas/ações de prevenção de disseminação dos microrganismos uma vez que, a prática do profissional está norteada na segurança do paciente, visando à redução do risco de danos associados à assistência à saúde. Assim, surgiu a questão: Quais são os fatores de risco de disseminação de microrganismos no exame de eletroencefalograma no serviço de neurologia diagnóstica?

Assim, objetiva-se avaliar os fatores de risco de disseminação de microrganismos relacionados com as dermatoses do couro cabeludo e, ainda, elaborar Procedimento Operacional Padrão (POP) e construir um E-book com POPs desenvolvidos em um SND de um hospital terciário do interior paulista, para elaboração de medidas preventivas e controle de infecção cruzada nos exames de EEG

\section{MÉTODO}

\section{Delineamento do estudo}

Trata-se de uma revisão integrativa de literatura com estratégia de busca em bases de dados virtuais, onde no âmbito da Prática Baseada em Evidências (PBE), é a integração entre a pesquisa científica e a prática profissional aplicada (22).

É um método de pesquisa que possibilita a síntese do estado do conhecimento pré-existente de uma determinada temática, identificando lacunas existentes para sugestões de novos estudos e perspectivas da temática estudada à partir de análises minuciosas (22-25).

Foram realizadas as seguintes etapas: elaboração da pergunta norteadora, busca ou amostragem na literatura, categorização e avaliação dos estudos, interpretação dos resultados dos estudos incluídos, discussão dos resultados e apresentação da revisão/síntese do conhecimento e foi utilizada a estratégia PICO à partir da questão norteadora já anteriormente citada. (23,26).

Em um primeiro momento do estudo, foi realizada uma revisão sobre o conhecimento a respeito de infecção cruzada relacionada às doenças do couro cabeludo. No segundo momento, surgiu uma preocupação sobre os fatores de risco aos quais estão sujeitos quando submetidos ao exame de EEG, suas medidas/ações para prevenção e controle de infecção cruzada.

Local de aplicação das medidas preventivas para controle de infecção cruzada

A aplicação das medidas preventivas foi realizada no SND localizado em um hospital terciário do interior paulista, público, universitário, que realiza 90 exames semanais, adulto e infantil, e que atende aproximadamente $90 \%$ do Sistema Único de Saúde (SUS).

\section{Estratégia para coleta de dados}

Os dados foram coletados durante os meses de janeiro e fevereiro de 2018 e para o levantamento dos mesmos, a busca ocorreu nas seguintes bases de dados: CINAHL (Cumulative Index to Nursing and Allied Health Literature), Embase
(Excerpta Medica), PubMed (U.S. National Library of Medicine), Scopus (SciVerse Scopus), e WoS (Web of Science).

Para estratégia de busca utilizou-se os seguintes descritores: "Dermatoses do Couro Cabeludo", "Eletroencefalograma”, "Desinfecção”, “Instituições de Saúde” e "Infecção Cruzada”, registrados no Descritores em Ciência da Saúde (DECS). Os critérios de inclusão foram os estudos que abordassem a temática da correlação entre o exame de EEG e infecção cruzada, em periódicos e textos disponíveis online na íntegra, nacionais e internacionais nos idiomas português, espanhol ou inglês, indexados e sem limitação temporal.

\section{Elaboração do POP e E-book}

A elaboração dos POPs seguiu as normativas estabelecidas pelo Núcleo de Gestão da Qualidade (NGQ) da instituição, norteada pela ISO 900I:2015 e pelo Manual Brasileiro de Acreditação (27-28). O E-book é o resultado que norteará com maior rapidez a equipe multiprofissional envolvida na realização do exame de eletroencefalograma do serviço de neurologia diagnóstica.

\section{RESULTADOS}

Foram identificados 936 artigos. A partir da exclusão daqueles que não contemplavam os objetivos do estudo e os duplicados, ao final, foram selecionados para análise, quatro estudos que estão apresentados no Quadro I.

Quadro I: Artigos selecionados a partir da revisão integrativa da literatura por base de dados online. 2019

\begin{tabular}{|c|c|c|}
\hline Bases de dados & $\begin{array}{c}\text { Número de artigos } \\
\text { recuperados }\end{array}$ & $\begin{array}{c}\text { Número de artigos } \\
\text { selecionados }\end{array}$ \\
\hline CINAHL & 205 & $\mathrm{I}$ \\
\hline Embase & 544 & $\mathrm{I}$ \\
\hline PubMed & 68 & 2 \\
\hline Scopus & 8 & 0 \\
\hline Web of Science & 111 & 0 \\
\hline Total & 936 & 4 \\
\hline
\end{tabular}

Os passos para a seleção dos artigos estão didaticamente apresentados no fluxograma abaixo:

Figura I: Fluxograma de recuperação e seleção dos artigos inseridos no estudo. 2018

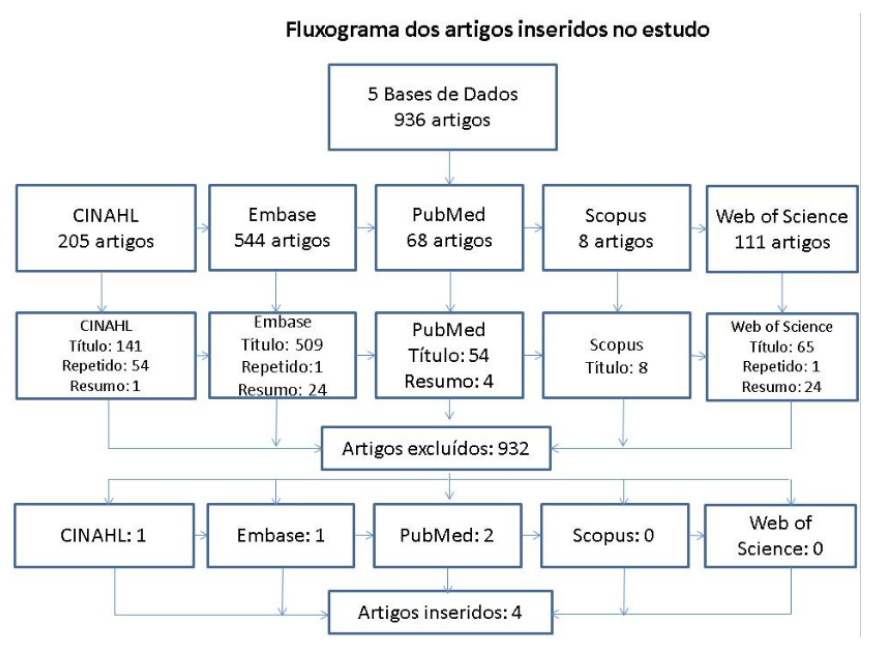


As caracterizações dos artigos selecionados estão resumidamente no Quadro 2.

Quadro 2. Instrumento para coleta de informações dos artigos selecionados. 2019

\begin{tabular}{|c|c|c|c|c|c|c|}
\hline Ref & $\begin{array}{c}\text { Base de } \\
\text { dados }\end{array}$ & Ano & Pais & Metodologia & Resultados & Conclusão \\
\hline 29 & PubMed & 2001 & França & $\begin{array}{l}\text { Realizado EEG } \\
\text { de indivíduos } \\
\text { que utilizam } \\
\text { eletrodos no } \\
\text { couro cabeludo } \\
\text { invasivo e não } \\
\text { invasivo. }\end{array}$ & $\begin{array}{l}\text { Abrasão não é } \\
\text { mais indicada } \\
\text { para realização } \\
\text { de EEG,devido } \\
\text { ao risco de } \\
\text { infecção e perda } \\
\text { da integridade } \\
\text { da pele. }\end{array}$ & $\begin{array}{l}\text { Indicado utilização } \\
\text { de eletrodos } \\
\text { descartáveis e } \\
\text { verificam que a } \\
\text { excelencia do EEG } \\
\text { pode ser obtido } \\
\text { sem abrasão no } \\
\text { couro cabeludo. } \\
\text { Devido ao risco } \\
\text { significativo } \\
\text { de infecção e } \\
\text { inconveniência } \\
\text { da lesão do } \\
\text { couro cabeludo.A } \\
\text { prática não é } \\
\text { mais aceitável em } \\
\text { pesquisa ou prática } \\
\text { clinica. }\end{array}$ \\
\hline 30 & PubMed & 2016 & França & $\begin{array}{l}\text { Pacientes } \\
\text { hospitalizados } \\
\text { em UTI com } \\
\text { resultado } \\
\text { positivo para } \\
\text { bactéria } \\
\text { multirresistente. }\end{array}$ & $\begin{array}{l}\text { Contaminação } \\
\text { cruzada por } \\
\text { klebisiella } \\
\text { pneumoniae } \\
\text { e possivel } \\
\text { contaminacao por } \\
\text { Acinetobacter } \\
\text { baumanni. }\end{array}$ & $\begin{array}{l}\text { O estudo não } \\
\text { permitiu afirmar } \\
\text { que a transmissão } \\
\text { cruzada se dá } \\
\text { pela realização } \\
\text { do exame, porém } \\
\text { após terem sido } \\
\text { adotadas medidas } \\
\text { de educação } \\
\text { e modifcação } \\
\text { do processo de } \\
\text { desinfecção dos } \\
\text { materiais, não } \\
\text { foram verificados } \\
\text { novos casos } \\
\text { de bactéria.A } \\
\text { Transmissão } \\
\text { cruzada através das } \\
\text { mãos não poderia } \\
\text { ser eliminada e o } \\
\text { uso de eletrodos } \\
\text { descartáveis } \\
\text { diminui o risco } \\
\text { de contaminação } \\
\text { cruzada. É } \\
\text { necessária limpeza } \\
\text { minuciosa para } \\
\text { prevenção. }\end{array}$ \\
\hline 31 & CINAHL & 2000 & Canadá & $\begin{array}{l}\text { Realizaram } \\
\text { exames com } \\
\text { eletrodos } \\
\text { intradérmicos, } \\
\text { entrevista e } \\
\text { coleta de exames } \\
\text { dos pacientes e } \\
\text { do profissional } \\
\text { que realizava o } \\
\text { EEG. }\end{array}$ & $\begin{array}{l}\text { Uso comum } \\
\text { de eletrodos } \\
\text { subcutâneos } \\
\text { foram os prováveis } \\
\text { veículos de } \\
\text { transmissão do } \\
\text { vírus. }\end{array}$ & $\begin{array}{l}\text { Procedimentos } \\
\text { de controle de } \\
\text { infecção foram } \\
\text { inadequados. } \\
\text { Hepatite B foi } \\
\text { resultado de } \\
\text { fonte comum de } \\
\text { infeç̧ão e práticas } \\
\text { inadequadas. }\end{array}$ \\
\hline 32 & Embase & 2013 & Londres & $\begin{array}{l}\text { Pacientes de } \\
\text { um hospital que } \\
\text { são suscetíveis } \\
\text { 'a infecção } \\
\text { por conta } \\
\text { das doenças } \\
\text { subjacentes e } \\
\text { intervenções. }\end{array}$ & $\begin{array}{l}\text { A superlotação,a } \\
\text { falta de higiene, } \\
\text { particularmente } \\
\text { a lavagem das } \\
\text { mãos, aumentam o } \\
\text { risco de infecção } \\
\text { cruzada. } \\
\text { A infecção } \\
\text { cruzada com vírus } \\
\text { transmitido pelo } \\
\text { sangue, como } \\
\text { hepatite b e c ou } \\
\text { HIV, é de grande } \\
\text { preocupação, mas } \\
\text { infelizmente é rara } \\
\text { devido às medidas } \\
\text { de controle. }\end{array}$ & $\begin{array}{l}\text { Os pacientes } \\
\text { são susceptiveis } \\
\text { a infecção por } \\
\text { devido doenças } \\
\text { subjacente e } \\
\text { intervenções } \\
\text { médicas, assim } \\
\text { como, sua } \\
\text { exposição a } \\
\text { microrganismos de } \\
\text { outros pacientes, } \\
\text { ambiente } \\
\text { hospitalar ou } \\
\text { pessoal do hospital. } \\
\text { As medidas para } \\
\text { prevenir a IRAS } \\
\text { são variadas e } \\
\text { lavagem das mãos } \\
\text { antes e depois do } \\
\text { procedimento ou } \\
\text { contato com cada } \\
\text { paciente. }\end{array}$ \\
\hline
\end{tabular}

\section{DISCUSSÃO}

Considerando o número significativo dos trabalhos identificados, acreditava-se que os artigos inseridos no estudo fossem superiores. Dentre os artigos selecionados, os autores abordam o controle de infecção como ponto prioritário na realização de exames de EEG, em que a colocação dos eletrodos pode causar lesão no couro cabeludo. Propõe medidas preventivas de educação, modificação no processo de desinfecção e controle de materiais utilizados.

O preparo para cuidar de pacientes, ocorre fortemente durante $o$ treinamento no próprio local de trabalho. A equipe de enfermagem deve estar devidamente capacitada para realização das técnicas de elaboração do exame, acompanhar - preparo da higienização do couro cabeludo para garantir a qualidade dos registros eletroencefalográficos, além de adotar medidas preventivas no controle de infecção, sendo a HM antes e depois do procedimento uma técnica de prevenção de IRAS, realizam também um importante papel na equipe multidisciplinar, abordando um trabalho educativo, cuidados assistenciais que atendam às necessidades específicas e individuais do paciente ${ }^{(29,33)}$.

Dentre os riscos de infecção na realização do EEG, a abrasão que é realizada com o intuito de atingir níveis de impedância, os eletrodos e sua fixação ficam susceptiveis de entrar em contato com a pele e derivados do sangue ${ }^{(29)}$.

Com esta preocupação, o centro de controle de doenças (CDC) emitiu orientações para desinfecção e esterilização de instrumentos reutilizáveis. Os instrumentos que tocam a pele íntegra, não são críticos e deve ser realizada desinfecção de baixo nível ou desinfecção intermediária. Os instrumentos que tocam membranas, mucosas, mas não vão tocar osso ou penetrar o tecido, são considerados semicríticos e devem ser sujeitos a desinfecção de alto nível, se não tiverem condições de serem esterilizados ${ }^{(34)}$.

Pela abrasão do couro cabeludo poder entrar em contato com derivados do sangue, é recomendada, então, a esterilização dos materiais expostos. Infelizmente, mesmo a desinfecção dos eletrodos do EEG é apenas superficial na maioria das pesquisas e laboratórios clínicos ${ }^{(29)}$.

Considerando tais colocações acima são enfáticos em relação ao tipo de desinfecção a ser feita, principalmente aquelas de alto nível, embora não especifiquem nenhum agente eficaz nesse processo, sugerindo até a esterilização dos instrumentos utilizados. A abrasão do couro cabeludo não é mais indicada para a realização do $E E G$, devido ao risco de infecção e pela perda da integridade da pele. Há uma preocupação sobre o erro de medição, quando a abrasão não era realizada, o que hoje é facilmente resolvido por meio da compreensão da relação entre impedância do couro cabeludo e eletrodo com a impedância dos amplificadores diferenciais modernos, podendo-se alcançar excelência de EEG. Um estudo na França, ainda propõe o descarte de material para impedir a infecção cruzada de bactérias multirresistentes, bactérias essas que foram identificadas em pacientes internados em Unidades de Terapia Intensiva (UTI) (29-30).

Uma pesquisa realizada no Canadá com o objetivo de determinar a extensão e fonte de surto de Hepatite B em uma clínica de EEG identificou que o uso comum de eletrodos subcutâneos foi o provável veículo de transmissão do vírus. Sugeriram a utilização de técnicas não invasivas para a realização do exame. Não sendo detectada nenhuma transmissão do vírus $\mathrm{HBV}$ quando foram utilizados eletrodos não invasivos. A transmissão da doença se deu por fontes como as mãos de profissionais, portanto, técnicas inadequadas, como não usar luvas, facilita a transmissão. Profissionais da saúde devem seguir as práticas de controle de infecção recomendadas e serem vacinados contra o vírus da Hepatite $B{ }^{(31)}$.

Essa investigação reforça a necessidade de padronização, 
não só da realização do exame, mas também do controle de desinfecção efetiva dos eletrodos e capacitação da equipe na manipulação de todos os utensílios necessária para o EEG, que vai de encontro com um estudo realizado em Londres com foco em IH.A utilização da ação preventiva é automática quando um sistema de gestão é baseado no risco ${ }^{(32,27)}$.

O aumento da vigilância por órgãos públicos e a obrigatoriedade da publicação das taxas de infecção aumentou o incentivo financeiro a essa questão, com o desenvolvimento de pacotes de cuidados para assegurar o cumprimento de diferentes medidas de controle ${ }^{(32)}$.

Os equipamentos de EEG requerem uma desinfecção eficaz para garantir a segurança do paciente e evitar risco de infecção cruzada conforme descritivo do equipamento de EEG registrado na ANVISA. A necessidade de limpar as superfícies dos componentes do sistema com um pano macio umedecido com sabão neutro, água ou etanol, em seguida um pano seco macio ou gaze. Para remover a pasta condutora, limpar imediatamente com pano úmido antes que o mesmo seque. Para limpeza do equipamento, limpe os componentes do sistema com um pano macio umedecido com desinfetante (35).

Para isso, podem ser usados a solução Gluconato de clorexidina $0,5 \%$, solução de cloreto de benzoténio $0,2 \%$, solução de glutaraldeido $2,0 \%$, cloreto de benzalconio $0,2 \%$, cloridrato alquil aminoetil glycine $0,5 \%$, phtharal $0,55 \%$ e fenol I,56\% (35).

A solução utilizada de álcool $70 \%$ tem ação bactericida, principalmente contra os vírus lipídicos. Indicado para desinfecção de instrumento não critico, semicrítico e superfícies. Pouco tóxico para os profissionais, pode causar ressecamento de pele, sendo recomendado o uso de luvas para o seu manuseio. $O$ fato de ser volátil e inflamável justifica ser estocado em recipiente fechado(36) o qual é utilizado no local de aplicação das medidas preventivas para o controle de infecção cruzada.

O uso de desinfecção com álcool etílico a $70 \%$ pode danificar instrumentos, dilatar e endurecer borrachas e certos plásticos.A eficácia e efetividade do álcool para desinfecção de materiais semicríticos alerta para padronização de métodos de aplicação do álcool e análise de complexidade estrutural do material a ser desinfetado, fatores estes que podem comprometer sua ação germicida ${ }^{(36)}$.

A SOBECC (Associação Brasileira de Enfermagem de CC, Recuperação Anestésica e Central de Material e Esterilização) descreve ainda que desinfetantes de nível quaternário de amônia apresentam como característica a baixa toxicidade e alta compatibilidade. As novas formulações de quaternário, de última geração, resultam em produto com espectro de ação ampliado(36).

A comissão controle de infecção relacionada à assistência a saúde (CCIRAS) da instituição proponente deste estudo, indica a utilização de quaternário de amônia, que é amplamente utilizado como desinfetante, tendo como vantagem adicional ser um produto com capacidade de remover sujidade em razão de sua ação transoativa. A atividade microbicida é atribuída à inativação de enzimas, desnaturação de proteínas celulares e ruptura de membrana celular. Apresenta ação bactericida, fungicida e virucida contra o vírus lipídico. Não tem ação esporocida, tuberculicida e virucida contra vírus não lipídico (37).

\section{CONCLUSÃO}

Foi possível identificar riscos de infecção cruzada no exame de EEG, a abrasão que é realizada no couro cabeludo para atingir níveis de impedância, eletrodos e suas extensões que entram em contato com a pele do paciente, a falta do produto padronizado utilizado na desinfecção do equipamento, a falta de materiais e eletrodos descartáveis utilizados no exame, a falta de controle de higienização das mãos, a falta de higienização do ambiente, a falta de treinamento com a equipe envolvida no processo de trabalho e a falta de protocolos a serem seguidas.

A higienização das mãos representa também uma evidência científica para a prevenção de IRAS e deve ser vista como medida primária para a prevenção e controle da disseminação da infecção hospitalar e tal prática está fortemente ligada às questões da segurança do paciente.

A educação em saúde é uma ferramenta que pode ser utilizada como métodos de promoção da saúde, prevenção de doenças de maneira individual e/ou coletiva, deve promover motivação e a capacitação para o autocuidado, assim como a compreensão da saúde como direito e responsabilidade pessoal e social.

Portanto, conclui-se que os riscos de adquirir infecções em serviços de saúde são elevados, que em grande parte poderiam ser evitados por meio da adesão dos profissionais da saúde às medidas/ações de prevenção e controle de infecção, garantindo assim a segurança do paciente. Tendo em vista a necessidade de implantação das medidas/ações de prevenção e controle de infecção elaborou-se mapeamento de processo, normas e rotinas, procedimento operacional padrão e indicadores no serviço de neurologia diagnóstica.

Os achados desta revisão integrativa evidenciam a necessidade de novos estudos que avaliem lesões de couro cabeludo e sua relação com infecções cruzadas, levando-se em conta a escassez de estudos que abordem o tema.

Por meio da evidência identificada pela revisão bibliográfica foi possível elaborar Mapeamento de Processo, Normas e Rotinas e Procedimento Operacional Padrão no SND de um hospital terciário do interior paulista.

\section{REFERÊNCIAS}

1. World Health Organization.The conceptual framework for the international classification for patient safety [Internet]. Geneva:WHO; 2009 [cited 2017 Aug 14]. Available from: http://www.who.int/patientsafety/taxo nomy/en/.

2. Brasil. Agência Nacional de Vigilância Sanitária. Assistência segura: uma reflexão teórica aplicada à prática. Brasília:Anvisa; 2017.

3. Runciman W, Hibbert P,Thomson R, SchaafTV, Sherman $H$, Lewalle P. Towards an international classification for patient safety: key concepts and terms. Int J Qual Health Care. 2009;2I (I): I8-26.

4. Medeiros EAS, Wey SB, Guerra C. Diretrizes para a prevenção e o controle de infecções relacionadas à assistência à saúde [Internet]. São Paulo: Comissão de Epidemiologia Hospitalar, Hospital São Paulo, Universidade Federal de São Paulo; 2005 [cited 2017 Aug 14]. Available from: http://www.apecih.org.br/ arquivos/Revista_APECIH.pdf

5. Joint Commission International. Patients as partners in the infection prevention and control process. Oakbrook Terrace, IL: Joint Commission International; 
2009.

6. Carmagnani MIS. Segurança e controle de infecção hospitalar. São Paulo: Reichmann e Afonso; 2000.

7. Brasil.Ministério da saúde.Portaria $n^{\circ} 529$, de $1^{\circ}$ de abril de 2013. Institui o Programa Nacional de Segurança do Paciente (PNSP). Diário Oficial da União [Internet]. 2 abr 2013 [cited 2017 Aug 14]. Available from: http:// bvsms.saude.gov.br/bvs/publicacoes/documento_ referencia_programa_nacional_seg uranca.pdf

8. Wenzel RP, Osternan CA. Hospital adquired infection surveillance in University hospital. Am J Epidemiol. 1987;103:253.

9. Boyce JM, Pittet D; Healthcare Infection Control Practices Advisory Committee; HICPAC/SHEA/APIC/ IDSA Hand Hygiene Task Force. Guidelines for hand hygiene in Health-care settings. Recommendations of the healthcare infection control practices advisory Committee and the HIPAC/SHEA/APIC/IDSA Hand Higyene task force. MMWR Recomm Rep. 2002;5 I (RR16): I-45.

10. Simmons, B. et al.The role of handwashing in prevention of endemic intensive care unit infections. Infect Control Hosp Epidemiol, v. I I, p.589-594, 1990.

11. Neves ZCP, Tipple AFV, Souza ACS, Pereira MS, Melo DS, Ferreira LR. Higienização das mãos: o impacto de estratégias de incentivo à adesão entre profissionais de saúde de uma unidade de terapia intensiva neonatal. Rev Lat Am Enfermagem [Internet]. 2006 [cited 2017 Aug 14];14(4):546-52. Available from: http://www. scielo.br/pdf/rlae/vl4n4/p t_vl4n4a I2.pdf

12. Lacerda RA. Controle de infecção em centro cirúrgico: fatos, mitos e controvérsias. São Paulo: Atheneu; 2003.

13. Souza AFL, Queiroz ACFLN, Oliveira LB, Valle ARMC, Moura MEB. Representações sociais da infecção comunitária por profissionais da atenção primária. Acta Paul Enferm. 2015;28(5):454-9. doi: http://dx.doi. org/I0.1590/I982-0194201500076.

14. São Paulo. Secretaria de Estado da Saúde. Infecção hospitalar: definições e conceitos. São Paulo: Sistema deVigilância Epidemiológica das Infecções Hospitalares do Estado de São Paulo; 2015.

15. Corrêa F, Lin K. Perfil epidemiológico dos exames de eletroencefalograma realizados no Hospital Universitário Prof. Polydoro Ernani de São Thiago (Florianópolis, SC) no ano de 2013. ACM Arq Catarin Med [Internet]. 2016; [cited 2018 Mar 4];45(3):35-47. Available from: http://www.acm.org.br/acm/seer/index. php/arquivos/article/view/109

16. Sabbatini RME. História do eletroencefalograma. Cerebro Mente [Internet]. 1997(3):[about 4 p.].

17. Carbofix Eacute. Brasília: Anvisa;13//2/2017.MS: 80005910019.

18. Guyton AC, Hall JE.Tratado de fisiologia médica. 12 ed. Rio de Janeiro: Elsevier; 201 I.

19. Pereira JM, Pereira FCN, Pereira VCN, Pereira IJN. Tratado das doenças dos cabelos e do couro cabeludo. Rio de Janeiro: DiLivros; 2016.

20. Conselho Federal de Enfermagem [Internet]. Brasília: Cofen; 2017 [cited 2017 Apr 20]. Available from: www. cofen.gov.br/resolução-cofen-3582009_4384.html

21. Manual do Técnico em EEG. São Paulo: Revinter; $2002-$ 2009. p.II9.
22. Pompeo DA, Rossi LA, Galvão CM. Revisão integrativa: etapa inicial do processo de validação de diagnóstico de enfermagem. Acta Paul Enferm. 2009;22(4):434-8.

23. Souza MT, Silva MD, Carvalho R. Revisão integrativa: o que é e como fazer. Einstein. 2010 [cited $2017 \mathrm{Apr}$ 22];8(I Ptl):102-6. Available from: http://www.scielo. br/pdf/eins/v8n I/pt_1679-4508-eins-8-I-0102.pdf

24. Mendes KDS, Silveira RCC, Galvão CM. Revisão integrativa:método de pesquisa para a incorporação de evidências na saúde e na enfermagem. Texto Contexto Enferm. 2008; 17(4):758-64.

25. Ganong LH. Integrative reviews of nursing research. Res Nurs Health. 1987; I0(I): I- I I.

26. Santos CMC, Pimenta CAM, Nobre MRC.A estratégia pico para a construção da pergunta de pesquisa e busca de evidencia. Rev Lat Am Enfermagem. 2007; 15 (3):508-II.

27. Lloyd's Register. ISSO 900I: 2015 - Certificação [internet]. São Paulo: LR; 2018[citado 13 Dez 2018]. Disponível em: www.Irqa.com.br/Certificacao/ISO900I-Sistemas-de-Gestão-da-Qualidade.

28. Couto R.C, Pedrosa TMG. Técnicas básicas para implantação da acreditação.Belo Horizonte: IAG Saúde; 2009. v. I.

29. Ferree TC, Luu PL, Russell GS, Tucker DM.ScalP electrode impedance,infection risk,and eeg data quality.Clin Neurophysiol. 200I Marc;1 I2(3):536-44. https://www.ncbi.nlm.nih.gov/pubmed/I I 222977

30. Weiss N, Faugeras F, Rohaut B, Leconte J, Lafeuille $\mathrm{E}$, Brossier $\mathrm{F}$, et al. Multidrug-resistant bacteria transmitted through high-density EEG in ICU. Seizure. 2016;37:65-8.

31. Johnson IL, Andonov A, Coulhart M, Carlson J, Chaudhary R, D'Cunha C, et al. An outbreak of hepatitis B associated with reusable subdermal electroencephalogram electrodes. CMAJ. 2000;162 (8):2217-3I. PMID: 10789626.

32. Breathnach AS. Nosocomial infections and infection control. Medicine. 20I3;4I (I I):649-53. doi: https://doi. org/l0.1016/j.mpmed.2013.08.010.

33. Diccini S. Enfermagem em neurologia e neurocirurgia. 2 ed. Rio de Janeiro:Atheneu; 2017.

34. Centers for Disease Control and prevention (CDC) [internet]. Infection control in dentistry. [citado 29 ago 2018]. Disponível em: http://www.cdc.gov.

35. Formulário de Petição para Cadastro de Equipamento. Disponível em: www4.anvisa.gov.br/base/visadoc/REL/ REL[40430-2-I5842].PDF.

36. Associação Brasileira de Enfermeiros de Centro Cirúrgico. Diretrizes de práticas em enfermagem cirúrgica e processamento de produtos para a saúde. SOBECC; 2017.

37. Brasil.Agência Nacional de Vigilância Sanitária. Portaria $n^{\circ} 15$, de 23 de agosto de 1988. Determina que o registro de produtos saneantes domissanitarios com finalidade antimicrobiana seja procedido de acordo com as normas regulamentares. Diário Oficial da União, de 05 de setembro de 1988. Disponível em: http://www.cvs. saude.sp.gov.br/zip/u_pt-anvisa-0I5_230888.pdf.

Recebido: 2019-09-24

Aceito: 2019-1I-22 